\title{
Prospective approaches for risk analysis in modern radiotherapy: the Italian experience and the contribution of medical physicists
}

\author{
L. Begnozzi ${ }^{1}$, M.C. Cantone ${ }^{2 a}$, B. Longobardi ${ }^{3}$ and I. Veronese ${ }^{2}$ \\ ${ }^{1}$ UOC di Fisica Sanitaria, Ospedale S. Giovanni Calibita Fatebenefratelli, Isola Tiberina, Via di Ponte Quattro Capi 39, 00186 Roma, Italy. \\ 2 Dipartimento di Fisica, Università di Milano,Via Celoria 16, 20133 Milano, Italy. \\ 3 Servizio di Fisica Sanitaria, Ospedale S. Raffaele, Via Olgettina 60, 20132 Milano, Italy.
}

Received 21 January 2013 - Accepted 3 June 2013

\begin{abstract}
In the last few years there has been significant development of radiation therapy (RT) equipment with advanced imaging and delivery techniques, as well as treatment planning systems. From this perspective, proactive approaches for risk assessment were identified as a powerful tool in modern radiation oncology. A multidisciplinary working group (WG) has been established in the framework of the Italian association for medical physics (AIFM) to promote the use of prospective approaches in the radiotherapy scientific community. This paper describes the main actions carried out by the WG in order to collect information about the engagement of Italian medical physicists in the risk management process, in reporting possible incidents in RT and in the procedures of collecting and analysing near misses. In particular, the main scope of the study was to evaluate the actual level of experience in use of proactive risk analysis tools in modern RT by medical physicists. Finally, the measures implemented by the WG in order to promote the use of such approaches, and consequently to contribute to enhancing safety and radiation protection culture in radiation oncology are described.
\end{abstract}

Keywords: radioprotection in radiotherapy / prospective approach for risk analysis

\section{Introduction}

The benefits of ionising radiation in medicine are well accepted, even though the risks coupled with its use cannot be entirely eliminated. While the diagnostic use of radiation requires suitable methodologies to minimise the dose without impairing the diagnostic quality (Hoeschen et al., 2010), the optimisation of patient protection in radiotherapy must be achieved by maintaining sufficiently high doses to irradiated tumours and protecting, at the same time, the healthy tissues to the largest extent (Mancosu et al., 2010).

In the last few years there has been significant development of radiation therapy (RT) equipment with advanced imaging and delivery techniques, as well as treatment planning systems with more complex image handling and dose calculation algorithms (Korreman et al., 2010; Reggiori et al., 2011). A common aspect of all the new technologies and methodologies introduced in modern RT is the level of complexity, evidently much higher than the recent past, i.e. before intensity-modulated radiation therapy (IMRT) became a major radiotherapy treatment modality.

The increased complexity related to the technological and process changes in RT places new demands on quality

\footnotetext{
a marie.cantone@unimi.it
}

assurance (QA) programmes, as well as innovative instrumentation and detectors for beam characterisation and checks (Veronese et al., 2010, 2013a, 2013b, 2013c). Moreover, new approaches to safety and radiation protection culture are required, since complexity may also increase the sensitivity to uncertainties and risk of accidental exposure (Amalberti, 2009). Examples of radiotherapy-related errors are unfortunately not uncommon, even in countries with the highest level of healthcare resources (Ash, 2007; Williams, 2007; Derremaux, 2008; WHO, 2008). In the light of these events, new challenges in radiation protection of patients are being faced at national and international levels, and measures are being taken to improve safety in radiotherapy treatments (Bourguignon, 2009).

In order to fully assess and manage the risks of accidental exposure deriving from the use of innovative radiotherapy methodologies, retrospective approaches, based on the lessons learned from events with major consequences, do not appear to be completely adequate, since they have the intrinsic limitation of being confined to the reported experiences, thus leaving unreported events or latent risks unaddressed. Similarly, the traditional approach to QA, based on guidelines with a list of tests and tolerance values for measured parameters, even though still valid, are difficult to fulfil in practice due to the increase in complexity of the RT chain. Therefore, prospective 
approaches, widely applied in high-risk industries, have to be implemented to find out the elements that could go wrong and identify, a priori, all the potential hazards that might occur during a complex RT treatment (Ford et al., 2009; Sawant et al., 2010; Ciocca et al., 2012; Perks et al., 2012).

Proactive approaches for risk assessment were identified as a powerful tool in modern radiation oncology by the Task group 100 of the American association of physicists in medicine (AAPM) (Huq et al., 2008). Their use was also recommended by the International commission on radiological protection (ICRP) as a resource for improving the safety of patients undergoing modern radiation therapy treatments (ICRP, 2009).

On the basis of these new needs, at the beginning of 2010 a multidisciplinary working group (WG) was established in the framework of the Italian association for medical physics (AIFM) to promote the use of prospective approaches in the radiotherapy scientific community.

The idea of establishing a working group was not motivated by a particular concern for patient safety related to some local or national adverse events, but by attention to the intrinsic complexity of new RT technologies; together with the consideration of the importance in contributing to broadening the ground of prevention of incidents and accidents in this field.

This manuscript describes the main actions carried out by the WG in order to collect actual and real information, from the different health structures, about aspects such as the engagement of Italian medical physicists in the risk management process, in reporting possible incidents in RT and in the procedures of collecting and analysing near misses. In particular, the main scope of the study was to evaluate the actual level of experience in use of proactive risk analysis tools in modern RT by medical physicists. Finally, the measures implemented by the WG in order to promote the use of such approaches, and consequently to contribute to enhancing safety and radiation protection culture in radiation oncology are described.

\section{The survey}

To improve knowledge of the Italian situation and the involvement of medical physicists in risk evaluation activities, in particular with the use of prospective approaches in advanced radiotherapy techniques, the WG carried out an investigation. A short questionnaire was distributed through the Italian association of medical physics to medical physicists employed in RT divisions of the various Italian health structures. The survey was performed in 2011, therefore the replies received show the situation in that year. The questions asked about:

1) "Does the risk management structure in your hospital actively involve physicists? In what way?"

2) "Are you aware of a method (model, form, procedure) that is followed in your heath structure for incident reporting in RT?"

3) "Are you aware of a method for collecting, analysing and evaluating data on near misses in RT in your health structure?"

4) "Are you aware of any other radiotherapy risk analysis activities (failure mode and effects analysis (FMEA), etc.)

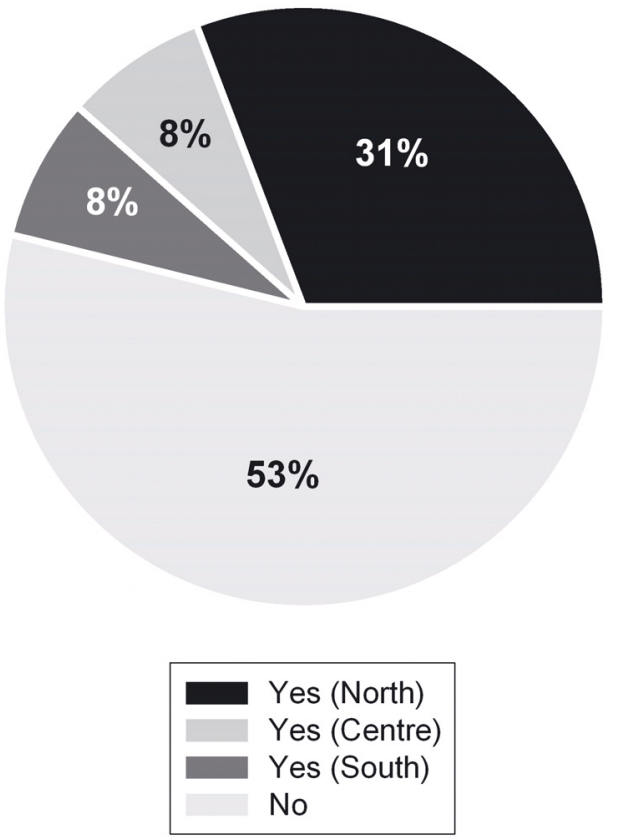

Fig. 1. Results, in percentage at national level, of the answers to the first question of the survey (1st question: does the risk management structure in your hospital actively involve physicists?).

being used in your health structure above and beyond normal quality controls?"

Even though the topic of this investigation was about the risks related to modern RT techniques, the questionnaire was sent to all the RT community, approximately 140 health structures: $40 \%$ of them located in the North of Italy (i.e. Aosta Valley, Emilia Romagna, Friuli Venezia Giulia, Liguria, Lombardy, Piedmont, Trentino Alto Adige and Veneto), 26\% in the Centre (i.e. Lazio, Marche, Tuscany and Umbria) and $34 \%$ in the South (i.e. Abruzzo, Apulia, Basilicata, Calabria, Campania, Sardinia and Sicily).

Thirty-nine centres from all over Italy replied to the questionnaire: 23 from the North, 10 from the Centre and 6 from the South. Despite the scarce data, it is possible to have an indication of the actual situation. The results of the investigation are shown in Figures 1-4.

Figure 1 shows the results of the first question. Approximately half of the medical physicists who took part in the survey answered that they were involved in risk management activities in their hospitals. Most of those involved work in the North (i.e. $31 \%$ ), while in the rest of Italy the participation of medical physicists was significantly lower (i.e. $8 \%$ in the Centre and $8 \%$ in the South).

It must be pointed out that in almost all Italian hospitals an Operative unit of risk management is (or is being) established, as recommended at a regional level. Generally, this unit is supported by the various complex structures of the hospitals, as well as by the general affairs, the legal affairs and the service for continuous quality improvement. The specific role of the medical physicists who were involved in risk management activities proved to be different according to the particular health structure. Indeed, in some cases the medical physicist (the director of the medical physics department or another appointed 


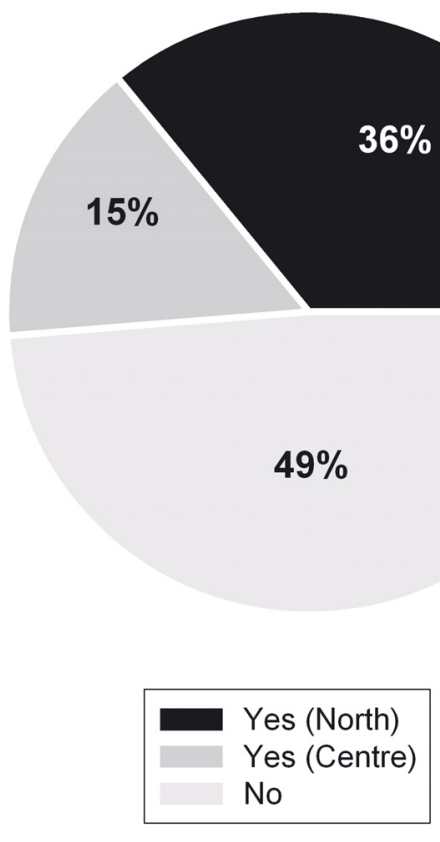

Fig. 2. Results, in percentage at national level, of the answers to the second question of the survey (2nd question: are you aware of a method (model, form, procedure) that is followed in your heath structure for incident reporting in RT?).

member of the department) is directly a full member of the Operative unit of risk management. In other cases, the medical physicist can temporarily be engaged by the unit for specific activities of risk management, in virtue of his competence and skill.

The results obtained from the second question were very similar to those of the first question, as shown in Figure 2. Indeed, in about half of the Italian centres the medical physicists are informed on procedures for collecting documentation on incidents in radiotherapy. What emerges is that most of the hospitals where incident reporting in RT has been organised are in the North (i.e. $36 \%$ of the total data collected), and the others are in the Centre (i.e. 15\%), while in the South there appears to be no documentation method for incident reporting, as far as medical physicists know.

Actually, in almost all Italian hospitals a policy for incident reporting is established, or is in a phase of fulfilment. Such a policy, which is common for all types of clinical risks and not specifically related to RT, consists of an internal procedure in the hospital for reporting adverse events occurring in clinical practice, through the use of dedicated informatics tools or forms. Considering the specific aspect of incident reporting in RT, the analysis of the results of the survey showed that the modality of reporting may be very different from one health structure to another. In most cases, the general procedure or form implemented by the hospital for reporting possible clinical incidents includes a section specifically related to RT. In other cases, a dedicated procedure for incident reporting is implemented directly by the department of radiation therapy, with the support of the medical physicists. In such cases, the procedure is not limited to the reporting stage, but periodic collegial analysis of the reports is organised in order to evaluate and

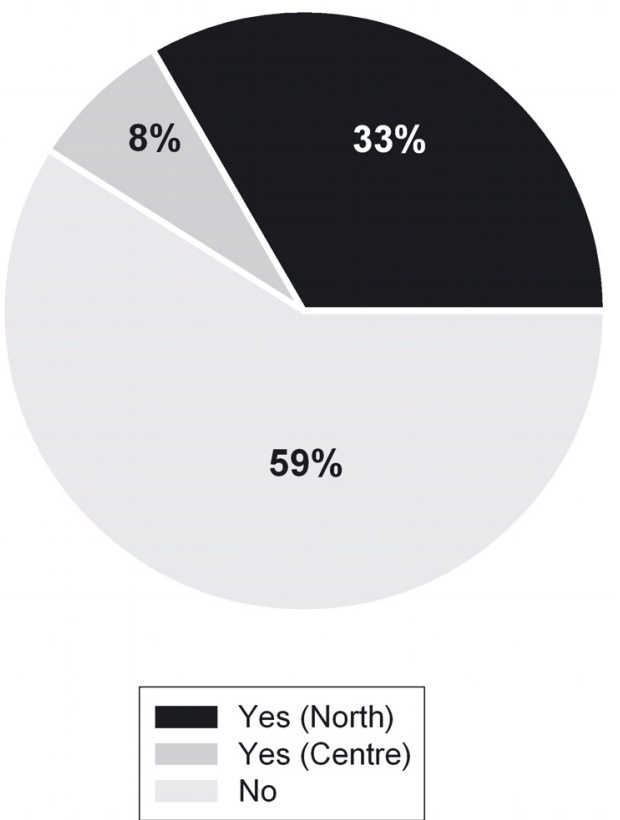

Fig. 3. Results, in percentage at national level, of the answers to the third question of the surve. (3rd question: are you aware of a method for collecting, analysing and evaluating data on near misses in RT in your health structure?).

study the causes of incidents and to implement, when needed, the proper corrective actions.

As can reasonably be expected, the level of attention for near miss reporting proved to be lower than incident reporting, as observed by the analysis of the data of the third question. Indeed, the percentage of Italian hospitals taking part in the survey where there is no data collection method for near misses in radiotherapy is equal to approximately $60 \%$, as shown in Figure 3. Most of the hospitals answering in the positive on the presence of data collection organisation are in the North, and the remainder in the Centre, while in the South there appears to be no documentation method for near miss reporting.

Finally, Figure 4 shows the results of the fourth question, about the knowledge and use of proactive methods for risk analysis in modern RT. The percentage of Italian hospitals taking part in the survey where the answer to the questionnaire indicated some specific prospective radiotherapy risk analysis activity was equal to only $21 \%(13 \%$ in the North and $8 \%$ in the Centre). This means that almost $80 \%$ of Italian centres have no prospective instruments for analysing and monitoring risk in radiotherapy. Therefore, it seemed clear that there was a need to make sector operators aware of risk management culture and prepare them for it.

\section{The actions of the working group}

On the basis of this situation, the WG started to promote the use of proactive methods for risk analysis in RT: workshops and courses were organised with the participation of radiation oncologists, medical physicists, technicians of radiology and representatives of technological research to discuss 


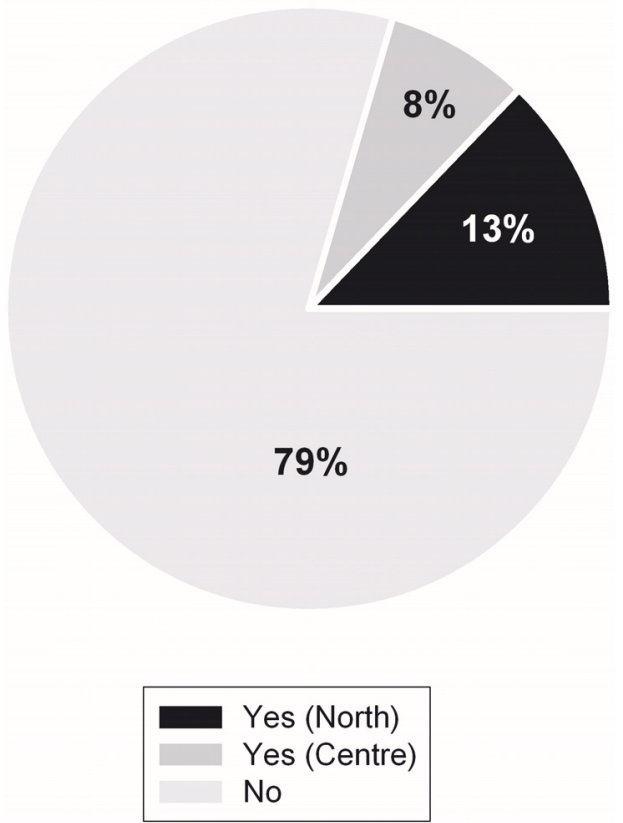

Fig. 4. Results, in percentage at national level, of the answers to the fourth question of the survey (4th question: are you aware of any other radiotherapy risk analysis activities (failure mode and effects analysis (FMEA), etc.) being used in your health structure above and beyond normal quality controls?).

the different aspects of new approaches in quality management. A report, drawn up by the members of the WG, dealing with the various aspects and methodologies for risk analysis and management in modern RT was also published by the AIFM (Begnozzi et al., 2012).

Furthermore, members of the WG promoted and supported various scientific studies, carried out in different health structures, with the aim of applying the failure mode and effects analysis (FMEA) approach to specific radiotherapy treatments. To date, investigations have been performed on electron beam intraoperative radiation therapy (IORT) delivered using mobile linear accelerators (Ciocca et al., 2012), on the proton beam radiotherapy process (Cantone et al., 2013), and on tomotherapy (Broggi et al., 2013).

On the basis of the results obtained in these studies and of the information accrued by the WG, a discussion was opened up within the AIFM WG regarding the available resources and the need for improving patient safety, since new technologies require a new focus on a multifaceted risk reduction strategy (proactive risk analysis, staff training-education-qualification, innovation in technologies preventing errors, effective communication and cooperation between disciplines, etc.). In particular, it was noticed that, although the retrospective approach is not fully effective for risk assessment in the new RT technologies, at the same time only poor information is available on the effectiveness of the proactive approach by itself: this fact seems to suggest and to direct one towards a form of integration between the two approaches. It means that proactive methods are not meant to replace retrospective methods, but rather the strength of these approaches resides in the synergy between them to improve overall safety in radiotherapy practice.

\section{References}

Amalberti R. (2009) The impact of new technologies on the risk accident, Controle 85, 65-66.

Ash D. (2007) Lessons from Epinal, Clin. Oncol. 19, 614-615.

Begnozzi L., Cantone M.C., Ciocca M., Longobardi B., Molinelli S., Pellegrini C., Sartor G., Soriani A., Spiazzi L., Tremolada V., Veronese I., Vitolo V. (2012) Approccio prospettico alla sicurezza del paziente nella moderna RT, Report AIFM $n^{\circ} 8$.

Bourguignon M. (2009) Radiotherapy: meeting the challenge of radiation protection for patients, Controle 185, 7-11.

Broggi S., Cantone M.C., Chiara A., Di Muzio N., Longobardi B., Mangili P., Veronese I. (2013) Application of failure mode and effects analysis (FMEA) to pretreatment phases in tomotherapy, J. Appl. Clin. Med. Phys. 14, 265-277.

Cantone M.C., Ciocca M., Dionisi F., Fossati P., Lorentini S., Krengli M., Molinelli S., Orecchia R., Schwarz M., Veronese I., Vitolo V. (2013) Application of failure mode and effects analysis to treatment planning in scanned proton beam radiotherapy, Radiat. Oncol. 8, 127.

Ciocca M., Cantone M.C., Veronese I., Cattani F., Petroli G., Molinelli S., Vitolo V., Orecchia R. (2012) Application of failure mode and effect analysis to intraoperative radiation therapy using mobile electron linear accelerators, Int. J. Radiat. Oncol. Biol. Phys. 82, e305-e311.

Derreumaux S., Etard C., Huet C., Trompier F., Clairand I., BottollierDepois J.F., Aubert B., Gourmelon P. (2008) Lessons from recent accidents in radiation therapy in France, Radiat. Prot. Dosim. 131, 130-135.

Ford E.C., Gaudette R., Myers L., Vanderver B., Engineer L., Zellars R., Song D.Y., Wong J., DeWeese T.L. (2009) Evaluation of safety in radiation oncology setting using failure mode and effects analysis, Int. J. Radiat. Oncol. Biol. Phys. 74, 852-858.

Hoeschen C., Mattsson S., Cantone M.C., Mikuz M., Lacasta C., Ebel G., Clinthorne N., Giussani A. (2010) Minimising activity and dose with enhanced image quality by radiopharmaceutical administrations, Radiat. Prot. Dosim. 139, 250-253.

Huq M.S., Fraass B.A., Dunscombe P.B., Gibbons J.P., Ibbott G.S., Medin P.M., Mundt A., Mutic S., Palta J.R., Thomadsen B.R., Williamson J.F., Yorke E.D. (2008) A method for evaluating quality assurance needs in radiation therapy, Int. J. Radiat. Oncol. Biol. Phys. 71, S170-S173.

ICRP Publication 112 (2009) Preventing Accidental Exposures from New External Beam Radiation Therapy Technologies, Ann. ICRP 39, Elsevier.

Korreman S., Rasch C., McNair H., Verellen D., Oelfke U., Maingon P., Mijnheer B., Khoo V. (2010) The European Society of Therapeutic Radiology and Oncology-European Institute of Radiotherapy (ESTRO-EIR) report on 3D CT-based in-room image guidance systems: A practical and technical review and guide, Radiother. Oncol. 94, 129-144.

Mancosu P., Cozzi L., Fogliata A., Lattuada P., Reggiori G., Cantone M.C., Navarria P., Scorsetti M. (2010) Collimator angle influence on dose distribution optimization for vertebral metastases using volumetric modulated arc therapy, Med. Phys. 37, 4133-4137.

Perks J.R., Stanic S., Stern R.L., Henk B., Nelson M.S., Harse Mathai M., Purdy J., Valicenti R.K., Siefkin A.D., Chen A.M. (2012) Failure Mode and Effect Analysis for Delivery of Lung Stereotactic Body Radiation Therapy, Int. J. Radiat. Oncol. Biol. Phys. 83, 1324-1329. 
Reggiori G., Mancosu P., Tozzi A., Cantone M.C., Castiglioni S., Lattuada P., Lobefalo F., Cozzi L., Fogliata A., Navarria P., Scorsetti M. (2011) Cone beam CT pre- and post-daily treatment for assessing geometrical and dosimetric intrafraction variability during radiotherapy of prostate cancer, J. Applied Clinical Med. Phys. 12, 141-152.

Sawant A., Dieterich S., Svatos M., Keall P. (2010) Failure mode and effect analysis-based quality assurance for dynamic MLC tracking systems, Med. Phys. 37, 6466-6479.

Veronese I., Cantone M.C., Chiodini N., Coray A., Fasoli M., Lomax A., Mones E., Moretti F., Vedda A. (2010) Feasibility study for the use of cerium-doped silica fibres in proton therapy, Radiat. Meas. 45, 635-639.

Veronese I., Cantone M.C., Catalano M., Chiodini N., Fasoli M., Mancosu P., Mones E., Moretti F., Scorsetti M., Vedda A. (2013a)
Study of the radioluminescence spectra of doped silica optical fibre dosimeters for stem effect removal, J. Phys. D 46, 015101.

Veronese I., Cantone M.C., Chiodini N., Fasoli M., Mones E., Moretti F., Vedda A. (2013b) The influence of the stem effect in Eu-doped silica optical fibres, Radiat. Meas. 56, 316-319.

Veronese I., Cantone M.C., Chiodini N., De Mattia C., Fasoli M., Mones E., Vedda A. (2013c) Radioluminescence dosimetry by scintillating fiber optics: the open challenges, Proc. SPIE $\mathbf{8 8 5 2}$ (Hard X-Ray, Gamma-Ray, and Neutron Detector Physics XV), 88521L.

Williams M.V. (2007) Radiotherapy Near Misses, Incidents and Errors: Radiotherapy Incident at Glasgow, Clin. Oncol. 19, 1-3.

WHO (2008) Radiotherapy Risk Profile, Geneva: WHO/IER/ PSP/2008.12.

Cite this article as: L. Begnozzi, M.C. Cantone, B. Longobardi, I. Veronese. Prospective approaches for risk analysis in modern radiotherapy: the Italian experience and the contribution of medical physicists. Radioprotection 49(1), 43-47 (2014). 